http://jmscr.igmpublication.org/home/ ISSN (e)-2347-176x ISSN (p) 2455-0450 crossref DOI: https://dx.doi.org/10.18535/jmscr/v9i6.28

\author{
Dournal Of Medical Science And Clinical Research \\ IGM Publication \\ An official Publication of IGM Publication
}

\title{
Assessment of Acute Toxicity Profile of Hypo-Fractionated Radiotherapy in Locally Advanced Breast Cancer
}

\author{
Authors \\ Hemendra Mishra ${ }^{1}$, Simrandeep Singh ${ }^{2}$, Tarun Kumar ${ }^{3}$, K. T. Bhowmik ${ }^{4}$, Kapil Suri ${ }^{5}$, \\ Rajiv Sharma ${ }^{6}$, Jaspreet Kaur ${ }^{7}$, Vikas Yadav, Vikas Madholia ${ }^{8}$, Akhilesh Mishra ${ }^{10}$, \\ Rajesh Sethi $^{11}$, Biju Azariah ${ }^{12}$, Ratika Gupta ${ }^{13}$ \\ ${ }^{1}$ Junior Resident, Department of Radiotherapy, VMMC \& Safdarjung Hospital, New Delhi \\ ${ }^{2,3}$ Senior Resident, Department of Radiotherapy, VMMC \& Safdarjung Hospital, New Delhi \\ ${ }^{4,5,6}$ Senior Professor, Department of Radiotherapy, VMMC \& Safdarjung Hospital, New Delhi \\ ${ }^{7}$ Professor, Department of Radiotherapy, VMMC \& Safdarjung Hospital, New Delhi. \\ ${ }^{8,9,10}$ Associate Professor, Department of Radiotherapy, VMMC \& Safdarjung Hospital, New Delhi \\ ${ }^{11}$ Medical Officer, Department of Radiotherapy, VMMC \& Safdarjung Hospital, New Delhi \\ ${ }^{12,13}$ Assistant Professor, Department of Radiotherapy, VMMC \& Safdarjung Hospital, New Delhi \\ Corresponding Author \\ Tarun Kumar \\ Senior Resident, Department of Radiotherapy, VMMC \& Safdarjung Hospital, New Delhi, India
}

\section{Introduction}

Breast cancer is the second most common cancer in the world and, by far, the most frequent cancer among women with an estimated 1.67 million new cancer cases diagnosed in 2012 (25\% of all cancers). ${ }^{1}$ It is the most common cancer in women both in developed and developing regions with slightly more cases in developed regions than in developing regions. Incidence rates vary nearly four-fold across the world regions, with rates ranging from 27 per 100,000 in Middle Africa and Eastern Asia to 92per 100,000 in Northern America. In India, breast cancer was estimated to cause 6.1 deaths per 100,000 population in the year 2016. The survival rate decreased by 2.7 times for breast cancer, in case of detection at stage IV as against stage I. A total of 80,700 women died of cancer breast in 2016 in India.
The established risk factors of breast cancer include the following: Higher age, the age at which women bear the first child and nulliparous women early menarche and late menopause are established risk factors. Both elevated oestrogen as well as progesterone are important factors in increasing breast cancer risk. In short, hormones appear to hold the key to the understanding of breast cancer. Breast cancer is common in higher socio-economic groups. This is explained by the risk factor of higher age at first birth. Exposure to radiation and Oral contraceptives are also the contributory factors in the causation of breast cancer.

Locally advanced breast cancer (LABC) represents some of the most aggressive breast cancers. Although in USA only $10-20 \%$ of all breast cancer patients present as LABC, in India, 
$30-60 \%$ present as a LABC. ${ }^{4}$ LABC was initially commonly defined asbreast cancers that were inoperable at presentation and/or having an extremely poor survival with locoregional treatments alone. However, now LABC refers to large breast tumors $(>5 \mathrm{~cm})$ associated with either the skin or chest wall involvement or with fixed axillary lymph nodes or disease spread to ipsilateral internal mammary node or supraclavicular node.

Over the past two decades a consensus has been reached on multimodality therapy of LABC, i.e the combination of systemic therapy, surgery and radiotherapy. Although the optimal administration sequence has not been established through clinical trials, initial systemic treatment is believed to be advantageous as it can increase resection and conservation rates of breast without compromising survival outcomes.

Radiotherapy in breast cancer has undergone various changes from anterior photon beams in early days to tangential beams to modern conformal radiotherapy treatment techniques in current era. Traditionally 50 Gy in 25 fractions (\#) is a standard radiotherapy protocol for post mastectomy patients. ${ }^{6}$ Results of trials from Whelan et al, Owen et al and START Trial lists groups, in early breast cancer have established low $\alpha / \beta$ values in breast tumors and thus suggests use of hypo-fractionated radiotherapy.

Implementation of hypo-fractionated schedules in routine clinical practice is convenient for patients as it reduces the number of hospital visits and helpful in busy radiotherapy establishments. Hence, there is a need to evaluate safety and efficacy of hypo-fractionated in patients. However, most of the cases in India presentation advanced stage, which are not amenable to breast conservation approaches and mostly undergomastectomy.

Adjuvant radiotherapy (RT) is an important part of breast cancer management but the dose and fractionation schedules used are variable. A total of $50 \mathrm{~Gy}$ in 25 daily fractions delivered over 5 weeks is often considered the "standard" adjuvant RT prescription. ${ }^{12,13}$ Hypo-fractionated regimes such as 42.5 Gy in 16 daily fractions, 5 fractions per week or $40 \mathrm{~Gy}$ in 15 daily fractions, 5 fractions per week have proven to be equally effective and achieve similar or better cosmetic and normal tissue outcomes for both invasive and in- situ diseases when treating the regional nodes. Hypofractionation is more convenient for patients and less costly. However, certain patients at higher risk of radiation induced late effects may benefit from more extended fractionation schedule. ${ }^{14}$ In this study, attempt has been made to study the acute toxicity profile of hypofractionated radiotherapy in locally advanced breast cancer after mastectomy.

\section{Material \& Methods}

This is a prospective study done on 30eligiblefemalepatients who were between 18-70 years of age as per inclusion and exclusion criteria, received Neo Adjuvant Chemotherapy (NACT) as per protocol and underwent Modified Radical Mastectomy or Simple Mastectomy. After obtaining the written informed consent for the participation in the study at Department of Radiotherapy in V.M.M.C and Safdarjung Hospital, New Delhi, patients were taken up for the radiation therapy.

Patients who received NACT and underwent Modified Radical Mastectomy or Simple Mastectomy, were histologically proven case of locally advanced intra ductal carcinoma in unilateral breast without distant metastasis. Patient with normal cardiac function one chocardiogram and absence of any medical co-morbidities that precludes the use of chemotherapy, surgery or radiotherapy and KPS score of $>70$ were included in this study.

Patients were excluded who have bilateral intra ductal Breast cancer, any previously treated contra lateral breast carcinoma by radiationtherapy and Palpable or radiologically suspicious of contra lateral axillary, supraclavicular or internal mammary nodes. Patient with Paget's disease of 
nipple and having pregnancy and lactation were also excluded from this study.

As NACT, 4 cycles of Cyclophosphamide + Adriamycin 3 weekly and 4 cycles of taxanes 3 weekly were given, after that patient was sent for assessment of surgery in the department of surgical oncology. Patients with operable invasive breast cancer underwent Modified Radical Mastectomy or Simple Mastectomy. After fulfilling the inclusion criteria, patients were included in study. All baseline investigations including complete hemogram, kidney function test (KFT), liver function test (LFT), X-ray chest, 2D echocardiogram etc. were done. After 3 weeks of surgery, when surgical wounds were completely healed and patients general condition was good then adjuvant radiotherapy (40Gy/15\#/3weeks) was delivered with $2 \mathrm{D}$ technique on COBALT 60 using bilateral tangential fields for chest wall and direct anterior field for supraclavicular fossa.

Patient evaluation during and after the radiotherapy

During the radiation therapy patient was evaluated weekly for toxicity to radiation by CTCAE criteria version 5.0.

After completion of radiation therapy, patient was evaluated monthly till 3 months. RTOG ACUTE Radiation Morbidity criteria15 were used for skin toxicity in this study.

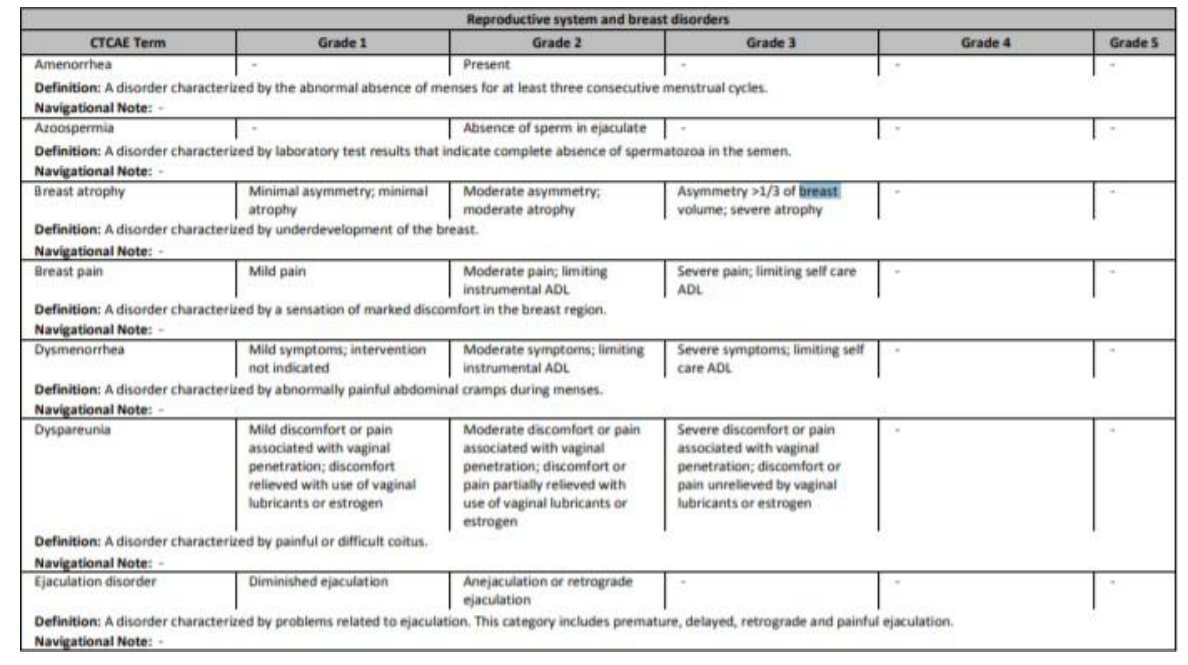

\section{Statistical Analysis}

Data analysis is done with the help of SPSS Software version 20. Comparison among study group was done with the help of appropriate statistical tests like unpaired t tests, paired t test, ANOVA etc. Qualitative data is presented with Frequency and Percentage tables, association among study parameters is assessed with the help of Chi-Square test. P value less than 0.05 is taken as significant level.

\section{Results}

This study showed that mean age of the participants was 45.4 years. Lump was the most common clinical feature in the study, and was present in $96.67 \%$ cases. Upper outer quadrant was the most common site of lump in breast i.e 13 (43.33\%) followed by central $8(26.67 \%)$. While weight loss was the least significant feature in the study patients. In this study 16 patients $(53.33 \%)$ were Post-menopausal, 11 (36.67\%) were Premenopausal and $3(10 \%)$ were Peri-menopausal respectively (table 1 ). 
Table 1:

\begin{tabular}{|c|c|c|}
\hline Demographic profile & No. of patients $(N=30)$ & Percentage \\
\hline \multicolumn{3}{|l|}{ Age in years } \\
\hline$\langle 31$ & 3 & 10 \\
\hline$\geq 31-50$ & 16 & 53.33 \\
\hline 251 & 11 & 36.67 \\
\hline \multicolumn{3}{|l|}{ Clinical Features } \\
\hline Lump & 29 & 96.67 \\
\hline Axillary lymphadenopathy & 9 & 30 \\
\hline Loss of appetite & 14 & 46.67 \\
\hline Discharge from the Nipple & 6 & 20 \\
\hline Weight loss & 5 & 16.67 \\
\hline \multicolumn{3}{|l|}{ Menopausal Status } \\
\hline Post-menopausal & 16 & 53.33 \\
\hline Pre-menopausal & 11 & 36.67 \\
\hline Peri-menopausal & 3 & 10.00 \\
\hline \multicolumn{3}{|l|}{ Quadrant of the breast } \\
\hline Upper outer & 13 & 43.33 \\
\hline Upperinner & 4 & 13.33 \\
\hline Lower outer & 3 & 10.00 \\
\hline Lower inner & 2 & 6.67 \\
\hline Central & 8 & 26.67 \\
\hline
\end{tabular}

Most common stage at the time of presentation was IIB $40 \%$ followed by III A with $30 \%$ of the patients. There were $5(16.66 \%)$ patients of II A and $4(13.33 \%)$ of stage III B in the study. There were no patients having stage I B or III C at the time of presenting to the hospital (table 2).

Table 2

\begin{tabular}{|l|c|c|}
\hline Stage at presentation & No. of patients & Percentage \\
\hline IIA & 5 & 16.67 \\
\hline IIB & 12 & 40.00 \\
\hline IIIA & 9 & 30 \\
\hline IIIB & 4 & 13.33 \\
\hline IIIC & 0 & 0.00 \\
\hline Total & $\mathbf{3 0}$ & $\mathbf{1 0 0 . 0 0}$ \\
\hline
\end{tabular}

Skin changes were the most common toxicity found in $10(33.33 \%)$ patients in this study. Grade 1 acute skin changes were found in 5 patients $(16.66 \%)$, Grade 2 skin changes were found in 3 patients $(10 \%)$ and Grade $3 \& 4$ found in 2 patients (6.66\%) respectively. Pain was the least common toxicity observed in $5(16.67 \%$ ) patients (table 3 ).

\section{Table 3}

\begin{tabular}{|c|c|c|c|}
\hline \multicolumn{2}{|l|}{ Toxicity } & No. of patients & Percentage \\
\hline \multicolumn{2}{|l|}{ Pain } & 5 & 16.67 \\
\hline \multirow{4}{*}{$\begin{array}{l}\text { Skin changes based on RTOG } \\
\text { criteria } \\
\qquad \mathrm{N}=10(33.33 \%)\end{array}$} & Grade 1 & 5 & $50 \%$ \\
\hline & Grade 2 & 3 & $30 \%$ \\
\hline & Grade 3 & 1 & $10 \%$ \\
\hline & Grade 4 & 1 & $10 \%$ \\
\hline \multirow[b]{3}{*}{ Subcutaneous fibrosis } & Grade 0 (None) & 22 & 73.33 \\
\hline & Grade 1 & 6 & 20 \\
\hline & Grade 2 & 2 & 6.67 \\
\hline \multicolumn{2}{|l|}{ Lymphedema } & 7 & 23.33 \\
\hline
\end{tabular}


Patients were followed up after 3 months of completion of radiation therapy. A complete physical examination along with routine blood investigations like Hemogram, KFT, LFT was done. If patients reported any complications, appropriate imaging like CECT, USG was performed. 29 (96.67\%) patients were disease free at 3 month follow up. 1 patient (3.33\%) had distant liver metastasis.

\section{Discussion}

The traditional standard radiotherapy schedule for breast cancer treatment delivers50 Gy total dose in 25 fractions daily over 5 weeks, 5 fractions per week. However, a lower total dose delivered in fewer, larger fractions (hypofractionation) is hypothesized to be as safe and effective as the standard treatment.

This study showed that mean age of participants was 45.4 years. This finding was comparable with the study of Bhattacharyya $\mathrm{M}$ et al. ${ }^{16}$ in which the mean age was 43.04 years. In a study by Mishra $\mathrm{R}$ et al ${ }^{17}$ with the age distribution $<31$ years $8 \%, 31$ 50 years $46 \%$ and the patients who were 51 years and above were $46 \%$. This finding was similar to present study. (Table 1)

In a retrospective comparative study of hypofractionated and conventional radiotherapy protocols in breast cancer patients by El-Sayed MI, Abdel-Wanis $\mathrm{ME}^{18}$ age at diagnosis was $<50$ years in $56.9 \%$ and $\geq 50$ years in rest $43.1 \%$ was similar to this study. In the study by Gogia A et al $^{19}$ the median age of the whole cohort was 47 years (range 23-72 years).

In the study by Bhattacharyya $\mathrm{M}$ et al. ${ }^{16}$ and Gogia A et al $^{19}$ pre-menopausal patients were $56 \%$ and $43 \%$ respectively, which was slightly higher as compared tothis study $36.67 \%$.

In a study by Mishra $\mathrm{R}$ et al the most common quadrant involved was Upper outer quadrant $44 \%$ followed by Central 25 (25\%) followed by Upper inner, Lower outer and Lower inner 16\%, 10\% and $5 \%$ which was more or less similar pattern as compared to this study.
Inaretrospectivecomparativestudyofhypofractionatedandconventionalradiotherapy protocols in breast cancer patients by El-Sayed 18

MI, Abdel-Wanis ME the proportions of patients for Stage I, Stage II and Stage III were 7.2\%, $61.9 \%$ and $30.9 \%$.

In the study by Bhattacharyya $\mathrm{M}$ et al. ${ }^{16}$ the most common stage of presentation was IIB 40\%, followed by IIIA $28 \%$, IIIB $12 \%$ and IIA $20 \%$ which was similar sort of sequence as present study.

In a retrospective comparative study of hypofractionated and conventional radiotherapy protocols in breast cancer patients by El-Sayed MI, Abdel-Wanis ME ${ }^{18}$ the skin changes (Grade 1 and 2 dermatitis) were noted in more than half of the patients $56.9 \%$ was more than present study $33.33 \%$. In this study, patients with hypofractionated radiation was safe and showed acceptable toxicity rate with only $10 \%$ incidence of grade II dermatitis. Moreover, it was statistically significant lower than in patients treated with conventional radiotherapy (10\% vs $25 \%, \mathrm{p}<0.0001)$. This is consistent with the combined results from the START A and START B trials, where a change in skin appearance occurred significantly less often in the hypofractionated radiation arm (39 Gy and 40 Gy arms) when compared with the 50 Gy arm (39 Gy HR0.6395\% CI0.47, 0.84, p=0.0019 and $40 \mathrm{~Gy}$ HR $0.7695 \%$ CI $0.60,0.97, p=0.0262)$. However, most trials reported that there was no difference in adverse events and toxicity between hypo-fractionated and conventional RT. The incidence of ischemic heart disease, symptomatic rib fracture and symptomatic lung fibrosis was low, with no differences between the study arms.

In the study by Bhattacharyya $\mathrm{M}$ et al. ${ }^{16}$ skin changes were found in $32 \%$ of the patients which was slightly lesser than this study and esophagitis in $16 \%$ of the patients, similar to this study which was $16.67 \%$. Also in this study, subcutaneous fibrosis in $28 \%$ patients more than present study which was $26 \%$ and lymphoedema $16 \%$ lesser to 
present study which was $23.33 \%$.

In the study by Abhilash GH et al. ${ }^{21}$ in hypofractionated arm delivered 39 Gy in 13 fractions, 5 fractions per week. Locoregional control was $86.7 \%$ with median follow up of 8 months. In present study at 3 month follow up, $96.67 \%$ patients had locoregional control.

\section{Conclusion}

Hypo-fractionated radiotherapy schedules is equally effective as standard protocol in terms of locoregional control, and in routine clinical practice is convenient for patients with manageable side effects as it reduces the number of hospital visits and helpful in high volume centers.

\section{References}

1. Latest world cancer statistics GLOBOCAN 2012: Estimated Cancer Incidence, Mortality and Prevalence Worldwide in 2012. 12 December 2013

2. Ferlay, Shin HR, Bray F, Forman D, Mathers C, Parkin DM. Estimates of worldwide burden of cancer in 2008. Globocan International journal of Cancer 2008; 127: 2893-2917.

3. WHO Bulletin (2018), Global Health Estimates 2016, Cancer, April2018.

4. Valero VV, Buzdar AU, Hortobagyi GN. Locally advanced breast cancer. Oncologist 1996;1:8-17.

5. Rakesh Chopra. The Indian scene of breast cancer. Journal of Clinical Oncology 2001;19:106-111 .

6. American College of Radiology. ACR Appropriateness Criteria®: postmastectomy radiotherapy. 2015:6.

7. Whelan TJ, Pignol JP, Levine MN, Julian JA, MacKenzie R, Parpia S, et al. Longterm results of hypofractionated radiation therapy for breast cancer. N Engl J Med 2010;362(6):513-20.

8. Owen JR, Ashton A, Bliss JM, Homewood $\mathrm{J}$, Harper C, Hanson J, et al. Effect of radiotherapy fraction size on tumor control in patients with early stage breast cancer after local tumor excision: long-term results of a randomized trial. Lancet Oncol 2006;7(6):467-71.

9. Bentzen SM, Agrawal RK, Aird EG, Barrett JM, Barrett-Lee PJ, Bliss J Metal. The UK Standardisation of Breast Radiotherapy (START) Trial A of radiotherapy hypofractionation for treatment of early breast cancer: a randomised trial. The Lancet. Oncology. 2008Apr;9(4):331-41.

10. Bentzen SM, Agrawal RK, Aird EG, Barrett JM, Barrett-Lee PJ, Bliss JM, etal. The UK Standardisation of Breast Radiotherapy (START) Trial B of radiotherapy hypofractionation for treatment of early breast cancer: a randomised trial. Lancet (London, England). 2008;371 (9618):1098-1107.

11. Haviland JS, Owen JR, Dewar JA, Agrawal RK, Barrett J, Barrett-Lee PJ, et al. The UK Standardisation of Breast Radiotherapy (START) trials of radiotherapy hypofractionation for treatment of early breast cancer: 10-year follow-up results of two randomized controlled trials. The lancet oncology. 2013Oct1;14(11):1086-94.

12. Adjuvant post mastectomy hypofractionated Radiotherapy in Egyptian cancer patients. Annals of Oncology 2012;23:34-36.

13. Boyle P, Ferday J. Epidemiology of breast cancer. Bailliere $S$ Clinical Oncology 1988;2:1 -57.

14. Thames HD, Bentzen SM, Turesson I, Overgaard M, Van den Bogaert W. Timedose factors in radiotherapy a review of human data. Radiotherapy and Oncology 1990;19:219-235.

15. Cox JD et al. Toxicity criteria of the Radiation Therapy Oncology Group (RTOG) and the European Organization 
for Research and Treatment of Cancer (EORTC). Int J Radiat Oncol Biol Phys. 1995 Mar 30;31(5):1341-6.

16. Bhattacharyya M, Kalita AK, Medhi PP, Jagtap V, Sunku R, Hassan FA, et al. Hypofractionated radiotherapy in post mastectomy locally advanced breast cancer: a study from a regional cancer center in North East India. Int J Res Med Sci 2018;6:3942-8.

17. Mishra R, Khurana R, Mishra H, Rastogi M, Hadi R Retrospective Analysis of Efficacy and Toxicity of Hypofractionated Radiotherapy in Breast Carcinoma Journal of Clinical and Diagnostic Research. 2016 Aug, Vol10(8):XC01-XC032

18. El-Sayed MI, Abdel-Wanis ME Comparison of hypofractionated and conventional radiotherapy protocols in breast cancer patients: a retrospective study. J Cancer Sci Ther.2012;4:158-63.

19. Gogia A, Raina V, Deo SV, Shukla NK, Mohanti BK, Sharma DN, Taxane and anthracycline Based Neoadjuvant Chemotherapy for Locally Advanced Breast Cancer : Institutional Experience; Asian Pacific Journal of Cancer Prevention, 2014:15 (5):1989-1992

20. Hopwood P, Haviland JS, Sumo G, Mills J, Bliss JM, et al. (2010) Comparison of patient-reported breast, arm, and shoulder symptoms and body image after radiotherapy for early breast cancer: 5-year follow-up in the randomised Standardization of Breast Radiotherapy (START) trials. Lancet Oncol 11:231-240.

21. Abhilash G. H, Anil Kumar Dhull, Rajeev Atri, Rakesh Dhankhar, Vivek Kaushal Comparison of hypofractionated radiation therapy versus conventional radiation therapy in post mastectomy breast cancer. Journal of Evidence Based Medicine and Healthcare, 2016 (26):1177-81. 